\title{
Inverse Symmetry Breaking in Multi-Scalar Field Theories $\ddagger$
}

\author{
Marcus Benghi Pinto ${ }^{1}$ and Rudnei O. Ramos ${ }^{2}$ \\ ${ }^{1}$ Departamento de Física, Universidade Federal de Santa Catarina, 88040-900 \\ Florianópolis, SC, Brazil \\ 2 Departamento de Física Teórica, Universidade do Estado do Rio de Janeiro, \\ 20550-013 Rio de Janeiro, RJ, Brazil \\ E-mail: marcus@fsc.ufsc.br, rudnei@uerj.br
}

\begin{abstract}
We review how the phenomena of inverse symmetry breaking (and symmetry nonrestoration) may arise in the context of relativistic as well as nonrelativistic multi-scalar field theories. We discuss how the consideration of thermal effects on the couplings produce different transition patterns for both theories. For the relativistic case, these effects allow the appearance of inverse symmetry breaking (and symmetry nonrestoration) at arbitrarily large temperatures. On the other hand, the same phenomena are suppressed in the nonrelativistic case, which is relevant for condensed matter physics. In this case, symmetry nonrestoration does not happen while inverse symmetry is allowed only to be followed by symmetry restoration characterizing a reentrant phase. The aim of this paper is to give more insight concerning the, qualitatively correct, results obtained by using one loop perturbation theory in the evaluation of thermal masses and couplings.
\end{abstract}

PACS numbers: $11.10 . \mathrm{Wx}, 98.80 . \mathrm{Cq}$

Submitted to: J. Phys. A: Math. Gen.

\section{Introduction}

Inverse symmetry breaking (ISB) is the name given to the phenomenon where a given symmetry may get broken at high temperatures. The possibility of such phenomenon taking place in the context of quantum field theory at finite temperature was first noticed by Weinberg [1] who also envisaged that a symmetry which is broken at zero temperature may not get restored at all at higher temperatures, a phenomenon called symmetry nonrestoration (SNR).

The possibility that some system may acquire lower symmetries as the temperature increases seems counter intuitive at first sight. However, there are plenty of real physical systems which do exhibit phenomena analogous to ISB/SNR. Some examples are given

$\ddagger$ Talk given by Marcus Benghi Pinto at QFEXT05, Barcelona, Spain, Sep. 5-9, 2005. 
by the Rochelle salt, liquid crystals, spin glass materials, compounds known as the manganites (e.g., ( $\left.\mathrm{Pr}, \mathrm{Ca}, \mathrm{Sr}) \mathrm{MnO}_{3}\right)$ and many other systems and materials, as recently reviewed in [2].

The idea of ISB/SNR has also called the attention of high energy physicists due to possibility of their implementation in realistic particle physics models, especially in the context of high temperature phase transitions in the early Universe [3, 4]. With this purpose, ISB/SNR have been used in applications covering problems which involve CP violation and baryogenesis, topological defect formation, inflation, etc [5].

The analysis of cosmological issues using condensed matter systems is a subject whose importance is growing lately as shown by research programmes such as the COSLAB (Cosmology in the Laboratory) [6]. With this purpose we have recently analyzed how ISB/SNR manifest themselves in nonrelativistic theories which may be used in condensed matter physics 4]. Our aim was to compare the finite temperature symmetry patterns of nonrelativistic models with the ones provided by previous studies concerning the relativistic case. As we shall review here, these patterns turn out to be completely different when the important thermal effects on the couplings are considered. We will review, in the next section, ISB/SNR issues concerning the relativistic model. Then, in section 3, we will examine the nonrelativistic case from a perturbatively point of view only. This type of analysis has not been performed in detail in Ref. [4] and will be included here since it gives new insights concerning the eventual breakdown of perturbation theory as well as the overall qualitative behavior of the symmetry patterns. Our conclusions are presented in section 4.

In the companion paper [7, we review the nonperturbative results for the general nonrelativistic model and present new results concerning an explicit application to dilute homogeneous binary Bose gases model $(U(1) \times U(1)$ BEC).

\section{Reviewing the original relativistic model}

The theory analyzed by Weinberg [1] consists of a $O\left(N_{\phi}\right) \times O\left(N_{\psi}\right)$ invariant relativistic model with two distinct types of scalar fields, $\phi$ and $\psi$, of $N_{\phi}$ and $N_{\psi}$ components, respectively. The interactions are mediated by a quadratic cross-coupling $\lambda$ as well as by self-interactions, $\lambda_{\phi}$ and $\lambda_{\psi}$. The Lagrangian density is given by

$$
\mathcal{L}=\frac{1}{2}\left(\partial_{\mu} \phi\right)^{2}-\frac{m_{\phi}^{2}}{2} \phi^{2}-\frac{\lambda_{\phi}}{4 !}\left(\phi^{2}\right)^{2}+\frac{1}{2}\left(\partial_{\mu} \psi\right)^{2}-\frac{m_{\psi}^{2}}{2} \psi^{2}-\frac{\lambda_{\psi}}{4 !}\left(\psi^{2}\right)^{2}-\frac{\lambda}{4} \phi^{2} \psi^{2},
$$

where $\phi^{2}=\sum_{i}^{N_{\phi}} \phi_{i} \phi_{i}$ and $\psi^{2}=\sum_{i}^{N_{\psi}} \psi_{i} \psi_{i}$. Overall boundness requires the couplings to satisfy $\lambda_{\phi}>0, \lambda_{\psi}>0$ and $\lambda_{\phi} \lambda_{\psi}>9 \lambda^{2}$. In the one-loop approximation we can readily compute the thermal masses for $\phi$ and $\psi$ with the results (at leading order in the high temperature expansion, $m_{\phi} / T, m_{\psi} / T \ll 1$ ] [1, [3]

$$
M_{i}^{2}(T) \simeq m_{i}^{2}+\frac{T^{2}}{12}\left[\lambda_{i} \frac{1}{2}\left(\frac{N_{i}+2}{3}\right)+\lambda \frac{N_{j}}{2}\right] \quad, \quad \text { where } \quad i, j=\phi, \psi .
$$


This equation shows how ISB/SNR can emerge for $\lambda<0$. For example, if one sets $m_{\phi}^{2}$ and $m_{\psi}^{2}$ positive to have a symmetric theory at $T=0$, ISB takes place by choosing

$$
|\lambda|>\frac{\lambda_{\phi}}{N_{\psi}}\left(\frac{N_{\phi}+2}{3}\right),
$$

which makes the $T^{2}$ coefficient of $M_{\phi}^{2}(T)$ negative while the same coefficient for $M_{\psi}^{2}(T)$ is kept positive, due to the boundness condition. In this case, high temperatures will induce the breaking $O\left(N_{\phi}\right) \times O\left(N_{\psi}\right) \rightarrow O\left(N_{\phi}-1\right) \times O\left(N_{\psi}\right)$ at the critical temperature

$$
\frac{T_{c}^{2}}{m_{\phi}^{2}}=24\left[|\lambda| N_{\psi}-\lambda_{\phi}\left(\frac{N_{\phi}+2}{3}\right)\right]^{-1} .
$$

On the other hand, when $m_{\phi}^{2}$ and $m_{\psi}^{2}$ are initially negative, $\lambda<0$ and Eq. (3) holds we have the emergence of SNR in the $\phi$ sector. At this point one could ask if ISB/SNR are not just artifacts of the naive one loop approximation. This is a rather important point since, in principle, higher orders could bring thermal effects to the couplings in such a way so as to suppress the phenomena. Let us consider the leading contribution, to the couplings, in the high temperature approximation

$$
\lambda_{i}(T) \simeq \lambda_{i}+\frac{3}{8 \pi^{2}} \ln \left(\frac{T}{M_{0}}\right)\left[\frac{1}{2}\left(\frac{N_{i}+8}{9}\right) \lambda_{i}^{2}+\frac{N_{j}}{2} \lambda^{2}\right]
$$

and

$$
\lambda(T) \simeq \lambda+\frac{\lambda}{8 \pi^{2}} \ln \left(\frac{T}{M_{0}}\right)\left[\frac{1}{2}\left(\frac{N_{\phi}+2}{3}\right) \lambda_{\phi}+\frac{1}{2}\left(\frac{N_{\psi}+2}{3}\right) \lambda_{\psi}+2 \lambda\right],
$$

where $M_{0}$ is a regularization mass scale. Then, the thermal masses can be rewritten as $M_{i}^{2}(T)=m_{i}^{2}+\left(T^{2} / 12\right) \Delta_{i}(T)$ where $\Delta_{i}(T)=\lambda_{i}(T)\left(N_{i}+2\right) / 6+\lambda(T) N_{j} / 2$.

It turns out that these improved results for $M_{i}^{2}(T)$ also allow for the appearance

of ISB/SNR [3, 8]. However, at this point one could raise an objection following the fact that those results are still perturbative and, as well known, perturbation theory eventually breaks down for field theories at finite temperatures. However, nonperturbative evaluations carried out with the Wilson Renormalization Group equations [8] as well as with the linear $\delta$ expansion [3] show the correctness of those results in the qualitative sense. Refs. 9] also support the occurrence of those exotic phenomena. That is, the inclusion of thermal effects on the couplings does not exclude the possibility of SNR/ISB occurring, at high temperatures, in $O\left(N_{\phi}\right) \times O\left(N_{\psi}\right)$ scalar relativistic models.

\section{The nonrelativistic model and the appearance of reentrant phases}

We now turn our attention to the analysis of SNR/ISB phenomena in the nonrelativistic limit. Let us first recall some fundamental differences between relativistic and nonrelativistic theories that will be important in our analysis. Firstly, the obvious reduction from Lorentz to Galilean invariance. Secondly, it should be noted that in 
the nonrelativistic description particle number is conserved and so, only complex fields are allowed. This second point will be particularly important to us since, for the processes entering the evaluation of the effective couplings only those that do not change particle number (the elastic processes) will be allowed. Another important difference between relativistic and nonrelativistic models concerns the structure of the respective propagators. While the relativistic propagator allows for both forward and backward particle propagation (which is associated to particles and anti-particles, respectively), the nonrelativistic propagator of scalar theories at $T=0$ only allows for forward propagation. Note however that the structure of the propagators in a thermal bath includes both backward and forward propagation [10]. The nonrelativistic lagrangian density is given by 4

$$
\begin{aligned}
\mathcal{L} & =\Phi^{*}\left(i \partial_{t}+\frac{1}{2 m_{\Phi}} \nabla^{2}\right) \Phi-\kappa_{\Phi} \Phi^{*} \Phi-\frac{g_{\Phi}}{3 !}\left(\Phi^{*} \Phi\right)^{2} \\
& +\Psi^{*}\left(i \partial_{t}+\frac{1}{2 m_{\Psi}} \nabla^{2}\right) \Psi-\kappa_{\Psi} \Psi^{*} \Psi-\frac{g_{\Psi}}{3 !}\left(\Psi^{*} \Psi\right)^{2}-g\left(\Phi^{*} \Phi\right)\left(\Psi^{*} \Psi\right) .
\end{aligned}
$$

The one body parameters $\kappa_{i}$, with $i=\Phi, \Psi$, can account for external potentials, or chemical potentials (in the grand canonical formalism) which is important for BoseEinstein condensation (BEC). The $m_{i}$ represents the (atomic) masses. For the BEC case, the couplings are related to the $s$-wave scattering length. Here, we will not attach any specific role to any of those parameters, leaving this to the companion paper [7]. The numerical factors and signs in the one and two-body potential terms have been chosen in such a way so that the potential is analogous the one considered in (11). The boundness condition for the model (17) is analogous to that of the relativistic model (11), requiring $g_{\Psi}>0, g_{\Phi}>0$ and $g_{\Psi} g_{\Phi}>9 g^{2}$. In addition, notice that for the nonrelativistic limit to be valid, one must keep $T \ll m_{i}$. In general, for nonrelativistic systems, the masses $m_{i}$ are of order of typical atomic masses, $m_{i} \sim \mathcal{O}(1-100) \mathrm{GeV}$. At the same time, the typical temperatures in condensed matter systems are at most of order of a few eV. Therefore, this condition will always hold for the ranges of temperature we will be interested in below.

For multi-component fields, (7) is a nonrelativistic multi-scalar model with symmetry $U\left(N_{\Phi}\right) \times U\left(N_{\Psi}\right)$ that is the analogue of the original relativistic model (11). For simplicity, in the following we set $N_{\Phi}=N_{\Psi}=1$, corresponding to an $U(1) \times U(1)$ symmetric model. In this case, one can write the complex fields in terms of real components as $\Phi=(1 / \sqrt{2})\left(\phi_{1}+i \phi_{2}\right)$ and $\Psi=(1 / \sqrt{2})\left(\psi_{1}+i \psi_{2}\right)$.

The nonrelativistic model, Eq. (17), falls in the same class of universality as the $O(2) \times O(2)$ relativistic case. At the one loop level, one can then write $\kappa_{i}$ in the high temperature approximation as

$$
\kappa_{i}(T) \simeq \kappa_{i}+\left(\frac{T}{2 \pi}\right)^{3 / 2} \zeta(3 / 2) \Delta_{i}^{\mathrm{NR}},
$$

defining the quantity $\Delta_{i}^{\mathrm{NR}}=(2 / 3) g_{i} m_{i}^{3 / 2}+g m_{j}^{3 / 2}$. 
Then, the critical temperature for symmetry restoration/breaking is

$$
T_{c, i}^{\mathrm{NR}}=2 \pi\left[\frac{-\kappa_{i}}{\Delta_{i}^{\mathrm{NR}} \zeta(3 / 2)}\right]^{2 / 3} .
$$

As in the relavistic case, the exotic transition patterns arise for $g<0$. For example, $\Delta^{\mathrm{NR}}$ signals the appearance of ISB or SNR in the $\Psi$ sector if one chooses $|g|>(2 / 3) g_{\Psi}$. We have seen that in the relativistic case this possibility survives to the inclusion of thermal effects on the couplings. However, as already discussed, the nonrelativistic contributions which are allowed in the computation of the four point Green's functions may produce $g_{i}(T)$ and $g(T)$ which differ drastically from the relativistic $\lambda_{i}(T)$ and $\lambda(T)$. In fact, the one loop evaluation, for high- $T$, gives [4]

$$
g_{i}(T) \simeq g_{i}-\frac{m T}{12 \pi} \sqrt{\frac{2 m}{\kappa}}\left(5 g_{i}^{2}+9 g^{2}\right)+\mathcal{O}(\kappa / T),
$$

and

$$
g(T) \simeq g-\frac{m T}{4 \pi} \sqrt{\frac{2 m}{\kappa}} g\left(g+\frac{2 g_{\Phi}}{3}+\frac{2 g_{\Psi}}{3}\right)+\mathcal{O}(\kappa / T),
$$

where we have set $m_{\Phi}=m_{\Psi}=m$ and $\kappa_{\Phi}=\kappa_{\Psi}=\kappa$. Note from the equations above that the effective couplings in the nonrelativistic theory have a much stronger dependence with the temperature than those in the equivalent relativistic theory. We therefore expect to see larger deviations at high temperatures for the effective couplings as compared with the same case in the relativistic problem (by high temperature we mean $\left.k_{i}(0)<<T<<m_{i}\right)$. It is also evident from the analysis of higher loop corrections to the effective couplings in the nonrelativistic model that all bubble like corrections contribute with the same power in temperature as the one-loop terms, which can easily be checked by simple power-counting in the momentum. A side effect of this is the breakdown, at high temperatures, of the simple one-loop perturbation theory applied here. Another symptom is the apparent running of the effective self-couplings, shown above, to negative values for sufficiently high temperatures given by $T^{\text {neg }} \sim$ $\min \left(12 \pi \sqrt{\kappa /\left(2 m^{3}\right)} g_{\Phi} /\left(5 g_{\Phi}^{2}+9 g^{2}\right), 12 \pi \sqrt{\kappa /\left(2 m^{3}\right)} g_{\Psi} /\left(5 g_{\Psi}^{2}+9 g^{2}\right)\right)$. At the same time, perturbation theory breaks down when the temperature dependent boundness condition, $R^{\mathrm{NR}}(T)=g_{\Psi}(T) g_{\Phi}(T) /[3 g(T)]^{2}$, becomes smaller than the unity. Nevertheless, it is easy to check that (for the parameters adopted below) the results obtained by just plugging Eqs. (10)-(11) above into (8) already shows a drastic qualitative difference between this simple improved approximation and the naive perturbative evaluation given by Eq. (8). This can be seen from figures 1 and 2 where $\kappa_{\Phi}(T)$ and $\kappa_{\Psi}(T)$ are displayed using temperature dependent and temperature independent couplings.

The parameter values are $g_{\Phi}(0)=8 \times 10^{-16} \mathrm{eV}^{-2}, g_{\Psi}(0)=7 \times 10^{-17} \mathrm{eV}^{-2}$, $g(0)=-5.5 \times 10^{-17} \mathrm{eV}^{-2}, m_{\Phi}=m_{\Psi}=1 \mathrm{GeV}$ and $\kappa_{\Psi}(0)=\kappa_{\Phi}(0)=1 \mathrm{neV}$. Using these values and Eqs. (10)-(11) in the evaluation of $\Delta^{N R}$ one sees the appearance of a reentrant phase on the $\phi$ sector characterized by ISB (at $T_{c}^{\mathrm{ISB}} \simeq 1 \times 10^{-3} \mathrm{eV}$ ) followed by $\mathrm{SR}$ (at $T_{c}^{\mathrm{SR}} \simeq 4 \times 10^{-3} \mathrm{eV}$ ). To assess the validity of perturbation theory one must be 


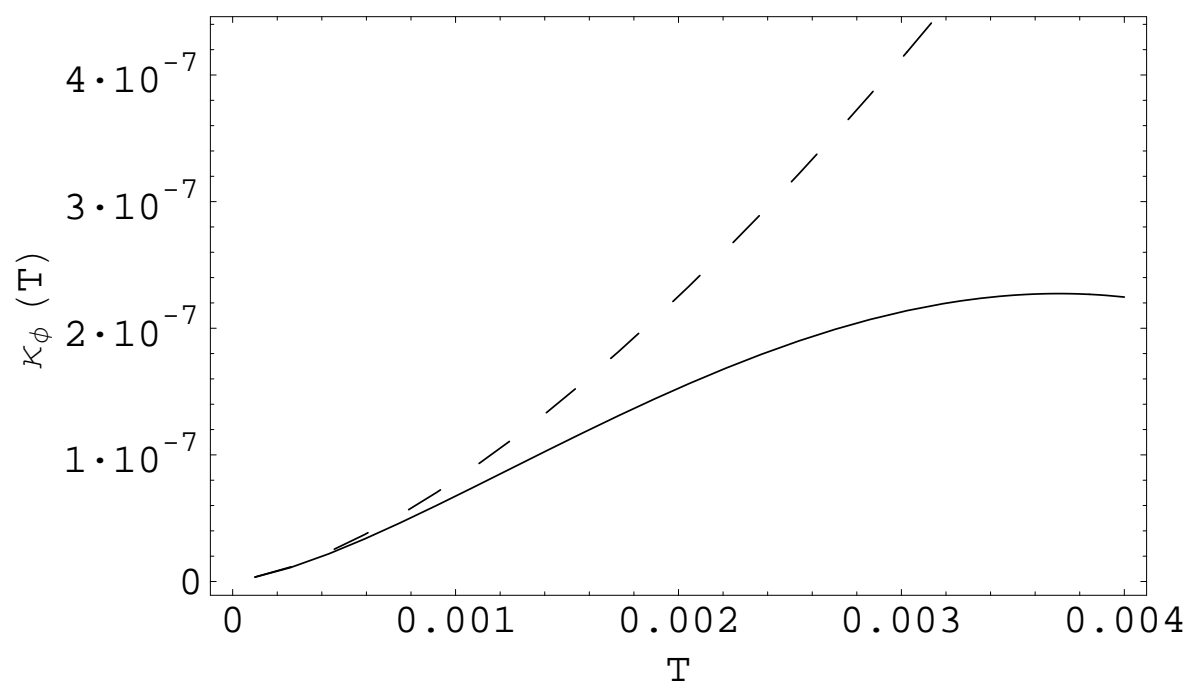

Figure 1. The quantity $\kappa_{\Phi}(T)$ obtained by using bare couplings (dashed line) as well as temperature dependent couplings (continuous line). Both, $\kappa_{\Phi}(T)$ and $T$ are given in $\mathrm{eV}$ unities.

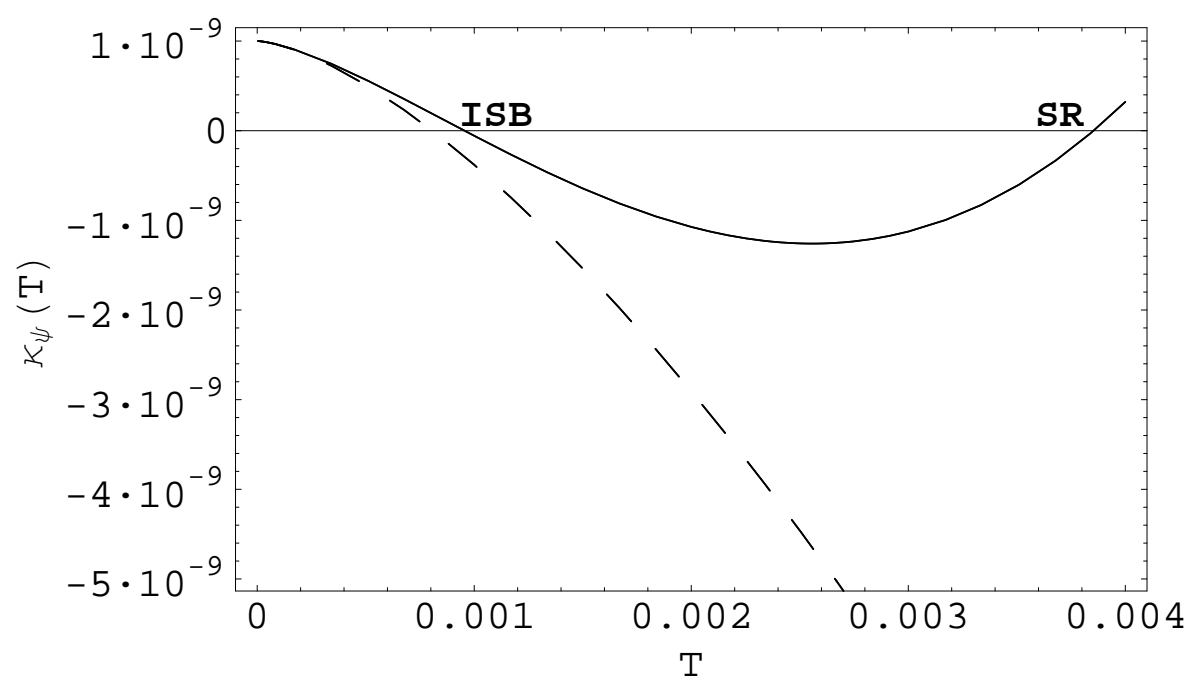

Figure 2. The quantity $\kappa_{\Psi}(T)$ obtained by using bare couplings (dashed line) as well as temperature dependent couplings (continuous line). In the second case one observes ISB followed by SR characterizing a reentrant phase. Both, $\kappa_{\Psi}(T)$ and $T$ are given in $\mathrm{eV}$ unities.

sure that those values of $T_{c}^{\mathrm{ISB}}$ and $T_{c}^{\mathrm{SR}}$ are smaller than the temperature which signals that the potential becomes unbounded via $g_{\Psi}(T)<0, g_{\Phi}(T)<0$ or $R^{\mathrm{NR}}(T)<1$. For the parameter values considered here, $g_{\Psi}(T)$ reaches negatives values, before $g_{\Phi}(T)$, at $T^{\text {neg }} \simeq 6.6 \times 10^{-3} \mathrm{eV}$. At the same time, figure 3 shows that $R^{\mathrm{NR}}(T) \leq 1$ at a smaller 
temperature, namely $T^{\text {unbound }} \simeq 4.675 \times 10^{-3} \mathrm{eV}$. Therefore, the appearance of the

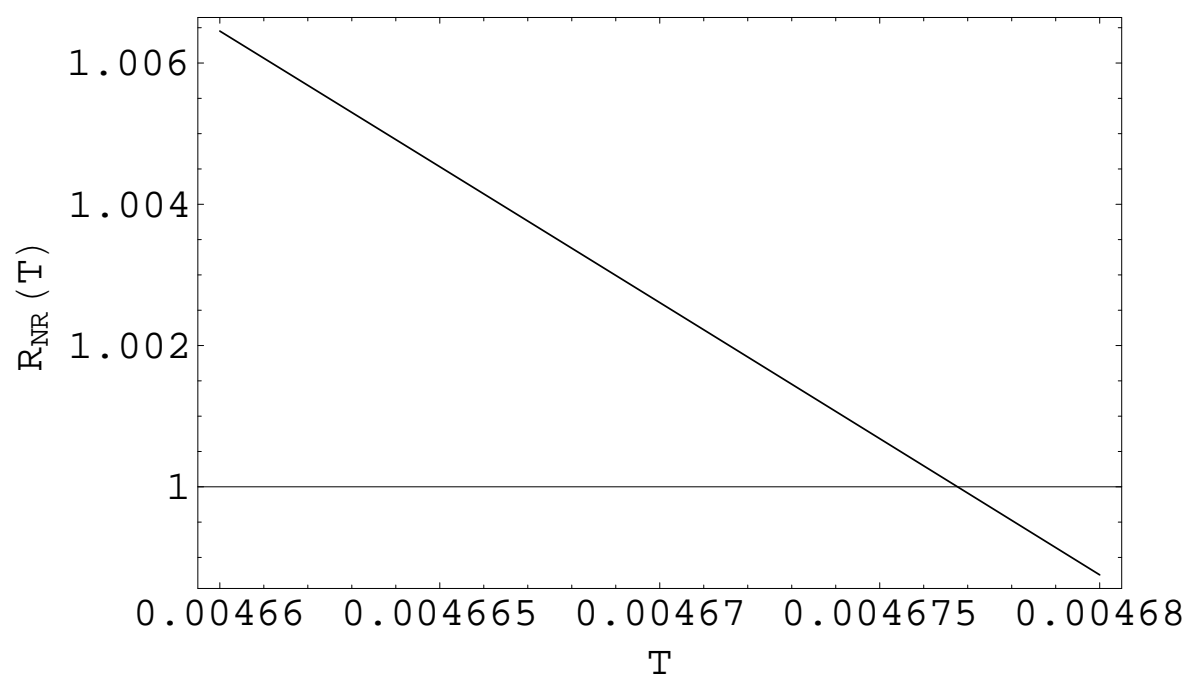

Figure 3. The dimensionless quantity $R^{\mathrm{NR}}(T)=g_{\Psi}(T) g_{\Phi}(T) /[3 g(T)]^{2}$ as a function of $\mathrm{T}(\mathrm{eV})$. One sees that $R^{\mathrm{NR}}(T) \leq 1$ at $T^{\text {unbound }} \simeq 4.675 \times 10^{-3} \mathrm{eV}$.

important reentrant phase, in the $\psi$ sector, happens at critical temperatures smaller than the ones which cause the instability of the potential. So, at least qualitatively, the strictly perturbative result presented in this paper is already rather satisfactory and has been confirmed by a nonperturbative resummation 4 .

The results presented here show that the phenomenon of reentrant phases, like those of ISB/SNR, observed in the relativistic models are not exclusively nonperturbative phenomena but also feasible within perturbation theory. Furthermore, reentrant phases in nonrelativistic models like (17) can be seen as a consequence of the interplay of the different energy scales available, that can be expressed in terms of $\kappa_{i}, m_{i}$ and the (dimensionfull) couplings, $g$ and $g_{i}$. At the same time, the relativistic model characterized only by the scales $m_{i}$, symmetry breaking/restoration phenomena is only accessible at high energy scales, $T \gg m_{i}$, as clearly shown by the general critical temperature result, Eq. (44), for perturbative values of coupling constants. The fact that the emergence of reentrant phases is a genuine feature of nonrelativistic models which is not affected by relativistic corrections can be seen, for instance, by considering the expansion of the relativistic dispersion relation

$$
\omega_{i}=\left(k^{2}+m_{i}^{2}\right)^{1 / 2} \sim m+\frac{k^{2}}{2 m_{i}}-\frac{1}{2 m_{i}}\left(\frac{k^{2}}{2 m_{i}}\right)^{2}+\ldots
$$

Since the temperature roughly gives the magnitude of the kinetic energy, the third term in (12) relative to the second one is of order $\mathcal{O}\left(T / m_{i}\right) \ll 1$. Higher order corrections to the nonrelativistic term are, therefore, negligible for the results obtained, e.g., for the reentrant phase temperatures, $T^{\mathrm{ISB}}$ and $T^{\mathrm{SR}}$, shown for instance in Fig. 2. 


\section{Conclusions}

We have seen how symmetry nonrestoration and inverse symmetry breaking may take place, at arbitrarily large temperatures, in multi-field scalar relativistic and nonrelativistic theories. These counter intuitive phenomena appear due to the fact that the crossed interaction can be negative while the models are still bounded from below. We have reviewed that, in the relativistic case, ISB/SNR survive the inclusion of thermal effects on the couplings. This qualitative result, first obtained perturbatively is confirmed by nonperturbative evaluations [3, 4, 8, 9]. Then, we have analyzed the possibility of obtaining such transition patterns in the nonrelativistic case. The naive use of temperature independent couplings allows ISB (and SNR) but the inclusion of the leading thermal contribution already causes of a drastic difference. Namely, ISB can show up in the nonrelativistic case only via the appearance of reentrant phases, as indeed observed in many real condensed matter systems. In the present work, our aim was to perform a deeper investigation of the perturbative results than the one which was done in Ref. [4. We have shown that perturbation theory is capable of predicting the right transition behavior provided one stays within its limit of applicability. Indeed, when thermal corrections are included, the qualitative results obtained here are verified by nonperturbative calculations as we review in Ref. [7] where the Bose-Einstein condensation problem for binary gases is considered.

\section{Acknowledgments}

The authors were partially supported by Conselho Nacional de Desenvolvimento Científico e Tecnológico (CNPq-Brazil). ROR was also partially supported by FAPERJ. We also would like to thank the organizers of the QFEXT05 workshop in Barcelona.

\section{References}

[1] Weinberg S 1974 Phys. Rev. D 93357

[2] Schupper N and Shnerb N M Preprint cond-mat/0502033

[3] Pinto M B and Ramos R O 2000 Phys. Rev. D 61125016

[4] Pinto M B , Ramos R O and Parreira J E 2005 Phys. Rev. D 71123519

[5] Mohapatra R N and Senjanovic G 1979 Phys. Rev. Lett. 42 1651; Dodelson S and Widrow L M 1990 Phys. Rev. Lett. 64, 340; Bajc B and Senjanović G 1997 Nucl. Phys. Proc. Suppl. A 52 246

[6] Rivers R J, Preprint cond-mat/0412404 published in: Proceedings of the National Workshop on Cosmological Phase Transitions and Topological Defects (Porto, Portugal, 2003), ed. Girard T A (Grafitese, Edificio Ciencia, 2004), 11-23.

[7] Ramos R O and Pinto M B, Symmetry Aspects in Nonrelativistic Multi-Scalar Field Models and Application to a Coupled Two-Species Dilute Bose Gas, in press J. Phys. A (2006).

[8] Roos T 1996 Phys. Rev. D 542944

[9] Jansen K and Laine M 1998 Phys. Lett. B 435 166; Bimonte G, Iñiguez D, Tarancón A and Ullod C L 1993 Nucl.Phys. B 559103

[10] Mahan G D Many-particle Physics (Plenum, New York, 1981). 